\title{
Comparison of solutions for microseismic focal mechanism estimation
}

\author{
Paweł Wandycz, Eryk Święch, Andrzej Pasternacki \\ AGH University of Science and Technology, Faculty of Geology, Geophysics and Environmental Protection, \\ Department of Fossil Fuel; al. A. Mickiewicza 30, 30-059 Krakow, Poland; \\ e-mail:pwandycz@agh.edu.pl,swiech.eryk@gmail.com,anpaster@agh.edu.pl
}

(C) 2016 Authors. This is an open access publication, which can be used, distributed and reproduced in any medium according to the Creative Commons CC-BY 4.0 License requiring that the original work has been properly cited.

One of the major advantages of microseismic data, recorded during hydraulic fracturing of prospective shale intervals is ability to use both $\mathrm{P}$ and $\mathrm{S}$ wave in the analysis, not only to determine epicentral locations of events but also to describe source itself. The information about the mechanisms of located microseismic events allows better understanding of in situ stress and strain conditions and the local subsurface geomechanical properties and forces (Kamei et al. 2015). As Duncan stated in his work, a proper characterization of the observed events mechanisms is the key to understand radiation pattern of the signals in the investigated area (Duncan \& Eisner 2010). Moreover, an understanding of the nature of the rock failure supports reservoir simulation models and stimulated reservoir volume estimates (Kratz \& Thorton 2016). Proper assessment of event strike, dip and rake provides the geometry of the fracture plane assuming double couple focal mechanism, while full moment tensor inversion provides information about shear and tensile nature of the calculated mechanisms.

The common method to obtain reliable focal mechanisms of observed microseismic events is decomposing of the full moment tensor. Seismic moment tensor is powerful tool which provides a general mathematical solution of sources that can be used to distinguish between various types of microseismic events. The method comes to reliably estimation of the six independent components of a full moment tensor by lestsquares inversion (Eaton \& Forouhideh 2010).

The motivation for this analysis was to determine microseismic focal mechanisms based on $\mathrm{P}$ wave peak amplitude, $\mathrm{P}$ and $\mathrm{S}$ - waves peak amplitudes and $\mathrm{S}$ - wave peak amplitude only to estimate the differences and uncertainties between these three different solutions. Furthermore authors decided to check how the mechanisms changes with different geometries of downhole monitoring array. In this study only synthetic data computed in $\mathrm{MiVu}$ GeoTomo software using raytracing method and simple layered velocity model were used. The mentioned velocity model was constructed based on well logs data delivered by PGNiG from measurements done in Northern Poland where active exploration of shale gas takes place. In this analysis authors focused only on double couple (DC) and compensated linear vector dipole (CLVD) mechanisms which are two most common types of microseismic focal mechanisms occur during hydraulic fracturing of shale deposits.

Performed analysis proved that the best and most consistent results with the lowest uncertainties reflected in the condition number parameter can be obtained by using both $P$ and $S$ peak amplitudes.

This study was supported by the Grant of The Polish National Center for Research and Development in the Program Blue Gas, project entitled "Appraisal of microseismic monitoring techniques of hydraulic fracturing and development of optimal processing 
and interpretation methodologies" (acronym: SHALEGASMICROS).

\section{REFERENCES}

Duncan P. \& Eisner L., 2010. Reservoir characterization using surface microseismic monitoring. Geophysics, 75, 5, 75A139-75A146.
Eaton D.W. \& Forouhideh F., 2010. Microseismic Moment Tensors: the Good, the Bad and the Ugly. CSEG Recorder, 35, 9, 44-47.

Kamei R., Nakata N. \& Lumley D., 2015. Introduction to microseismic source mechanisms. The Leading Edge, 34, 8, 876-878.

Kratz M. \& Thornton M., 2016. The effect of location error on microseismic mechanism estimation: synthetic and real field data examples. First Break, 34, 1, 55-59. 